\title{
Performance Analysis of OFDM based Cognitive Radio Network through Energy Detection
}

\author{
Jasleen Kaur \\ M.Tech Student DAVIET, Jalandhar \\ Dr. Suyeb.A.Khan \\ Associate Professor SSIET Patti \\ Dr.Jagjeet Malhotra \\ Associate Professor DAVIET, Jalandhar
}

\begin{abstract}
With the development of a new and ever expanding wireless applications and services, spectrum resources are facing in demands. In present scenario, the spectrum allotment has been done by providing each new service with its own fixed frequency Slot. Most of the user's spectrum is already assigned, so it becomes very difficult to find spectrum for other users or existing users. This leads to the scarcity of available spectrum and inefficient channel utilization. Cognitive radio is a novel technology which improves the spectrum utilization by allowing secondary user to borrow unused radio spectrum from primary licensed users or to share the spectrum with the primary users. Present paper deals with the spectrum sensing in which multiple users detect the spectrum gap through energy detection and investigate the detection performance in an efficient and implementable way. Simulation results showed that the probability of detection is achieved at small value SNR in case of OFDM modulation as compare the other and simple cognitive environment.
\end{abstract}

\section{INTRODUCTION}

Today's wireless networks are recognized by a static spectrum assignment policy. Recently, due to the demand of spectrum increases continuously, however, telecommunication services face the spectrum scarcity at particular spectrum bands. On the contrary, a large portion of the assigned spectrum is still used sporadically leading to under-utilization of the significant amount of spectrum [1]. The limited available spectrum and the inefficiency

in spectrum use make the requirement of new communication system to exploit the existing wireless spectrum opportunistically. Cognitive radio technology is one of the solution of these current spectrum inefficiency problems [2]. A cognitive radio is designed to be aware of and sensitive to the changes in its surrounding, which makes spectrum sensing an important requirement for the realization of cognitive radio networks. Spectrum sensing enables unlicensed users, to adapt to the environment by detecting unused spectrum portions without causing interference to the licensed network, referred to as the primary network. Demand for wireless services requires the use of more spectrum resources. However, today's wireless networks are characterized by a fixed spectrum assignment policy. As a result, few spectrum resources such as $2.4 \mathrm{GHz}$ unlicensed industrial, scientific, and medical (ISM) band are currently available for 
future wireless applications [1]. Operating in unlicensed band leads to create interference between systems users degrades the system performance. To make the intelligent wireless communication system, cognitive radio is aware of the radio frequency environment, selects the communication parameters (such as carrier frequency, modulation type, bandwidth and transmission power) to optimize the spectrum usage and adapts is transmission and reception accordingly [2]. By sensing and adapting to the environment, a cognitive radio is able to allot in the spectrum and serve its user without causing interference to the licensed user. To do so, the cognitive radio must continuously sense the spectrum it is using in order to detect the reappearance of the primary user. Once the primary is detected the cognitive radio should withdraw from the spectrum instantly so as to minimize the interference. This is very difficult task as the various primary users will be employed different modulation schemes, data rates and transmission powers in the presences of variable propagation environment and interference generated by other secondary users [3]. The probability of detection $\left(\mathrm{P}_{\mathrm{d}}\right)$ and the probability of false alarm are the parameters for the detection of performance of spectrum sensing. The probability of secondary user declares the presence of a PU when the spectrum is occupied by the PU is called the probability of detection $\left(\mathrm{P}_{\mathrm{d}}\right)$. The probability of presence of Primary user when the spectrum is idle is called the probability of false alarm. The probability of absence of primary user when the spectrum is occupied declares by secondary user called probability of miss detection $\left(\mathrm{P}_{\mathrm{m}}\right)$. Mathematically, probability of miss detection is $\mathrm{P}_{\mathrm{m}}=1-\mathrm{P}_{\mathrm{d}}$. The false alarm reduces spectral efficiency and miss detection causes interference with the PU. Generally, it is most important to detect maximum probability of detection in subject to minimum probability of false alarm.

\section{SPECTRUM SENSING TECHNIQUES}

The process of detection of primary user (license user) is called spectrum sensing. The spectrum sensing techniques is generally classified into three categories; Energy Detection, Matched Filter coherent detection, and Cyclostationary Feature Detection [4]. In [3], it shows that the matched filter can maximize the received SNR which will be mentioned, it requires demodulation of a primary user signal and it can perform coherent detection. The advantage of matched filter is that according to consistent, to get high processing gain less time is needed. However, a significant disadvantage of a matched filter is that a special receiver is necessary for every primary user. So the utilization of the matched filter is restricted by the drawback mentioned above.

The energy detector simplifies the matched filter to perform non-coherent detection. It detects the received signalse energy to compare with the threshold and then deduce the status of the primary signals. The disadvantage is that a threshold we used will be easily influenced by unknown or changing nose levels, so the energy detector will be confused by the presence of any in-band interference [3].

It is investigated that within a special modulation type, the cyclostationary feature detector is able to exploit the inherent periodicity in the received signal to detect primary signals since most signals vary with time periodically [14]. There is also a disadvantage that longer processing time and higher computational complexity is needed with the cyclostationary feather detector [11].

In [14], it indicated that all these methods above are depending on the transmitter detection. Through the spectrum sensing of CR users, they can deduce that whether there is a signal from 
primary transmitter which is present in a spectrum or not. Among the three techniques, the energy detector has been mostly widely used in radiometry. Since the energy detector has been mostly widely used in radiometry. So we only introduce the energy detector in details. The energy detector is able to be implemented as a spectrum analyzer, it takes the average frequency bins of a Fast Fourier Transform (FFT)[8]. Among them, energy detection has been widely used since it does not require any a priori knowledge of the primary signals and has much lower complexity than the other two schemes. In addition, it does not need any prior information about the PUs' signals [5].

The aim of the present paper is to comprehend the utilization of spectrum sensing in cognitive radio networks, and investigate the performance of the Energy Detection as a spectrum sensing Technique at different modulation. Matlab software is used to simulate the signals from the cognitive radio networks and an energy detector to determine the status of the primary users. After getting the result, try to draw the relationship between the factor SNR to and the final detections and investigate how the SNR influence the detections.

\section{SYSTEM MODEL}

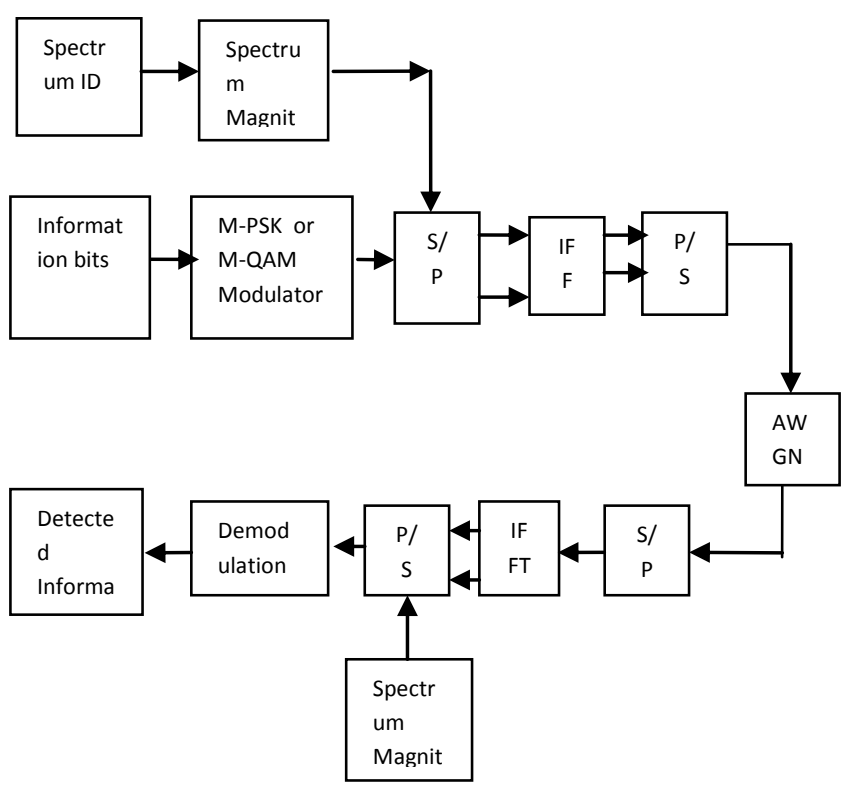

Figure 1 System Model

In CR network $K$ number of secondary users and primary users are considered with common receiver, as shown in Fig. 1[5]. It is assumed that each secondary user sensing the spectrum independently and then the local decisions are sent to the common receiver which can combine all the available decision to conclude the absence or presence of the primary users (PU). The essence of spectrum sensing is a binary hypothesis-testing problem. Consider there are $\mathrm{M}$ $>1$ number of antennas are present at the receiver. In the Eqn 3 the spectrum sensing at CR $i$ is considered [6]. The sensing method is to decide between the following two hypotheses, $\mathrm{X}(\mathrm{t})=\left[\mathrm{X}_{1}(\mathrm{t}) \ldots \ldots . \mathrm{X}_{\mathrm{M}}(\mathrm{t})\right]^{\mathrm{T}}$; 
$\mathrm{S}(\mathrm{t})=\left[\mathrm{S}_{1}(\mathrm{t}) \ldots \ldots . . \mathrm{S}_{\mathrm{M}}(\mathrm{t})\right]^{\mathrm{T}}$

$\mathrm{n}(\mathrm{k})=\left[\mathrm{n} 1(\mathrm{t})::: \mathrm{n}_{\mathrm{M}}(\mathrm{t})\right]^{\mathrm{T}}:$

$H_{0}:$ Absent the Primary user

$H_{1}$ : Present the Primary user

$$
\begin{aligned}
\left(\mathrm{X}_{\mathrm{i}} t\right)= & \{(t), & & H_{0} \\
& h(t) s(t)+\mathrm{n}_{i}(t), & & H_{1} \ldots(1)
\end{aligned}
$$

where $\mathrm{X}_{i}(t)$ is the received signal at the $i_{\text {th }}$ CR in time slot $t, s(t)$ is the primary user signal, $\mathrm{n}_{i}(t)$ is the additive white Gaussian noise (AWGN), and $h_{i}(t)$ denotes the complex channel gain of the sensing channel between the PU and the $\dot{\iota}_{\text {th }}$ CR. For a given probability of false alarm $\mathrm{P}_{\mathrm{fa}}$, i.e. the probability of mistaking the presence of the primary signal when it actually not present. The system model is to choose the hypothesis for maximizes the probability of detection $\mathrm{Pd}$, i.e. the probability of correctly determining the presence of the primary signal, for a given number of samples. In the description that follows, following [5-6] it would be shown that the optimal receiver derived from Neyman-Pearson theorem reduces to many known estimators. Special emphasis will be given to a few latest techniques in cooperative sensing, and one blind sensing algorithm will also be evaluated. For a given $\mathrm{P}_{\text {fa }}$, the Neyman-Pearson theorem states that $\mathrm{P}_{\mathrm{d}}$ will be maximized for the following decision statistic, which is essentially the likelihood ratio test (LRT):

$\mathrm{T}_{\mathrm{LRT}}(x)=\frac{p\left(x / H_{1}\right)}{p\left(x / H_{0}\right)} \ldots$

Where $X$ is the aggregation of $X(t) ; t=0 \ldots \ldots \ldots . . . N-1$ [7]. The model assumes that the distributions of signal and noise are known, which hardly the case is. Under the assumption that we are dealing with a flat fading channel, and the $\mathrm{s}_{\mathrm{i}}(\mathrm{k})$ 's are independent over $\mathrm{k}$, we have the following PDFs

$P((X \mid H i))=\prod_{k=0}^{N-1} p(X(k) \mid H i) \ldots(3)$

Assuming Gaussian distributions for noise and signal samples, i.e. $n(k) \sim N\left(0, \sigma_{n}^{2} I\right)$, and $s(k) \sim N\left(0, R_{n}\right)$ the LRT reduces to estimator-correlator (EC) detector

$T_{E C}(x)=\sum_{k=0}^{N-1} x^{T}(k) R_{s}\left(R_{s}+\sigma_{n}^{2} I\right)^{-1} x(k)$

It can further be noticed that $R_{s}\left(R_{s}+\sigma_{n}^{2} I\right)^{-1} x(k)$ is the MMSE estimation of $\mathrm{s}(\mathrm{k})$, so that TEC is actually the correlation of the observed signal $\mathrm{x}(\mathrm{k})$ with MMSE estimation of $\mathrm{s}(\mathrm{k})$. The assumption that $R_{s}=\sigma_{s}^{2}$ Ireduces $\mathrm{T}_{\mathrm{EC}}$ to the energy-detector (ED) given by

$T_{E D}(x)=\sum_{k=0}^{N=1} x^{T}(k) x(k)$

Furthermore, under the assumption that $\mathrm{s}(\mathrm{k})$ is deterministic and known to the receiver, LRT

reduces to the matched-filter detector given by

$T_{M F}(x)=\sum_{k=0}^{N-1} S^{T}(k) x(k)$

Volume 8 Issue 3 June 2017

ISSN: 2319-1058 
So, we notice that the LRT reduces to different known detectors under given constraints If prior knowledge of the PU signal is unknown, the energy detection method is optimal for detecting zero-mean constellation signals [7-8]. For the $i_{\text {th }}$ CR with the energy detector, the average probability of false alarm, the average probability of detection, and the average probability of missed detection over AWGN channels are given, respectively, by [11]

$$
\begin{aligned}
& P_{f, i}=\frac{\Gamma\left(u, \frac{g i}{2}\right)}{\Gamma(u)} \ldots .(4) \\
& P_{d, i}=Q_{u}(\sqrt{2 \gamma i}, \sqrt{\lambda i}) \ldots . .(5) \\
& P_{m, i}=1-P_{d, i} \quad \ldots . . .(6)
\end{aligned}
$$

In the above equations, $\lambda i$ and $\gamma i$ denote the energy detection threshold and the instantaneous signal-to-noise ratio (SNR) at the $i_{\text {th }} \mathrm{CR}$, respectively, $u$ is the time-bandwidth product of the energy detector.

$\Gamma(a, x)=\int_{x}^{\infty} t^{\alpha-1} e^{-t} d t$

$\Gamma(a, x)$ is the incomplete gamma function. $(a, x)$ is the generalized Marcum Q function $[9-10,12]$.

\section{IMPLEMENTATION OF OFDM \& OTHER MODULATION IN COGNITIVE ENVIRONMENT}

The CR is evaluated with MPSK at different value M for BPSK, QPSK and M-Ary on AWGN channel. The data stream is encoded using non return to zero NRZ encoding. In NRZ encoding the first thing is to keep the binary signal's amplitude of 1, where it has value on and replace value of ' 0 ' with ' -1 '. Secondly, the number of elements of binary signal are up sampled by repeating the number of 1's and number of -1's to make the size of matrix equal to the size of carrier wave over the specific time. Afterwards, the NRZ encoded data is multiplied by a carrier wave. This help act to make a 180 degree phase shift of the carrier wave, where -1 is present and a 0 degree phase shift where 1 is present

One of the main requirements of cognitive radio networks is the detection of primary users as quickly as possible. Orthogonal Frequency Division Multiplexing (OFDM) based cognitive radio networks are known to be an excellent fit for the architecture of cognitive radio networks $[11,12]$. Since, multi-carrier sensing can be exploited in OFDM-based networks the overall sensing time can be reduced. Once a primary user is detected in a single carrier, sensing in other carriers is not necessary. In [11], a power-based sensing algorithm in OFDM networks is proposed for spotting a primary user. It has been shown that the overall detection time is reduced by collecting information from each carrier. However, this necessitates the use of a large number of carriers, thereby increasing the design complexity. Hence, novel spectrum sensing techniques need to be developed such that the number of samples needed to detect the primary user is minimized within a given probability of detection error. The modulation scheme based on orthogonal frequency division multiplexing (OFDM) is a natural approach that might satisfy desired properties [16]. OFDM has become the modulation of choice in many broadband systems due to its inherent multiple access mechanism and simplicity in channel equalization, plus benefits of frequency diversity and coding [16]. OFDM is a multi-carrier modulation technique that can overcome many problems that arise with high bit-rate communications, the most serious 
of which is time dispersion. The data bearing symbol stream is split into several lower-rate streams, and these streams are transmitted on different carriers. Because this splitting increases the symbol duration by the number of orthogonally overlapping carriers (subcarriers), multipath echoes affect only a small portion of the neighbouring symbols. The orthogonality as illustrated in Figure 1 (system Model), of all modulated subcarriers is maintained if the subcarrier spacing equals the reciprocal value of the OFDM symbol duration and rectangular pulse shaping is assumed [14]. The cognitive engine is responsible for making intelligent decisions for primary and secondary users. The transmission opportunities are identified by the decision unit based on the information from the energy detection, as well as local and network spectrum sensing data.

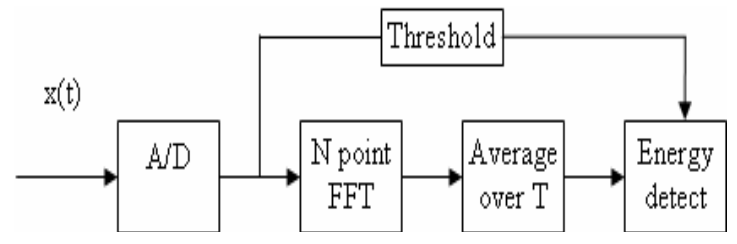

Figure 2 Energy Detection

\section{V. $\quad$ SIMULATION RESULT AND DISCUSSION}

The Simulation has been carried out for cognitive radio network on MATLAB under AWGN channel. Under this simulation five primary users and secondary users in the spectrum have been considered. The performance has been detected with the probability of detection, false alarm probability and missed probability under different number of SNR. The Energy detection is used to detect the vacant spectrum in cognitive radio network, where primary users are not present. The third primary user in is not available in the proposed cognitive network as shown in fig. 3 Immediately assigned this vacant spectrum to secondary users as shown in fig. 4 and whenever primary user want to occupy the spectrum, secondary user immediately left this vacant spectrum. The carrier frequencies $1000 \mathrm{~Hz}$ to $5000 \mathrm{~Hz}$ for five users are used and sampling frequency is $12000 \mathrm{~Hz}$. The Power spectrum density of signals are calculated and compared with the threshold value to determine the availability of primary users. The simulation is carried out for the analysis of detection probability under different number of SNR at $\mathrm{Pfa}=0.01$ and time bandwidth factor $u=100$ as shown in fig 5. SNR was varied between 0 to $100 \mathrm{~dB}$. It also shows that with the increasing of the SNR the detection also increased as shown in Table 1. Similarly, the detection probability is increased between $20 \mathrm{~dB}$ to $40 \mathrm{~dB}$ bit it almost constant or 1 after $40 \mathrm{~dB}$ to $100 \mathrm{~dB}$ as shown in fig 9. Fig. 6 shows that performance of missed detection varies based on SNR. It has been found that missed probability detection decreases from 0.8 to zero as SNR is varies from $0 \mathrm{~dB}$ to $100 \mathrm{~dB}$. The use of multicarrier OFDM in Cognitive network which result in minimize spectrum sensing error (i.e., sum of false alarm and miss detection probabilities) since minimizing spectrum sensing error both reduces collision probability with primary user and enhances usage level of vacant spectrum. This lead to provide reliable spectrum sensing performance (i.e., minimize spectrum sensing error), one of the great challenges is determining threshold level since spectrum sensing performance depends on the threshold level. When determining threshold level, besides spectrum sensing error, spectrum sensing constraint which requires false alarm and miss detection probabilities to be below target level should also 
be considered since it guarantees minimum required protection level of primary user and usage level of vacant spectrum.

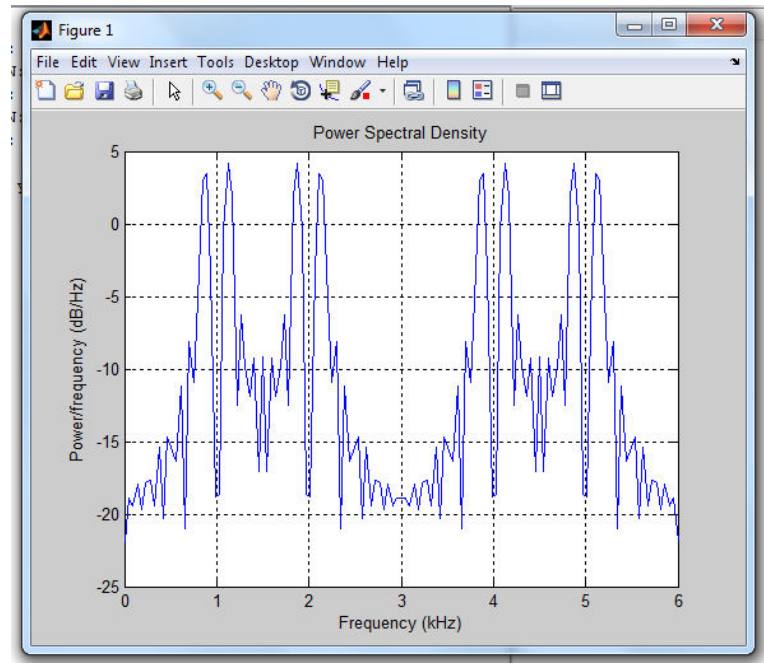

Figure 3 Spectral Efficiency of Four Primary Users where third Primarv user is absent

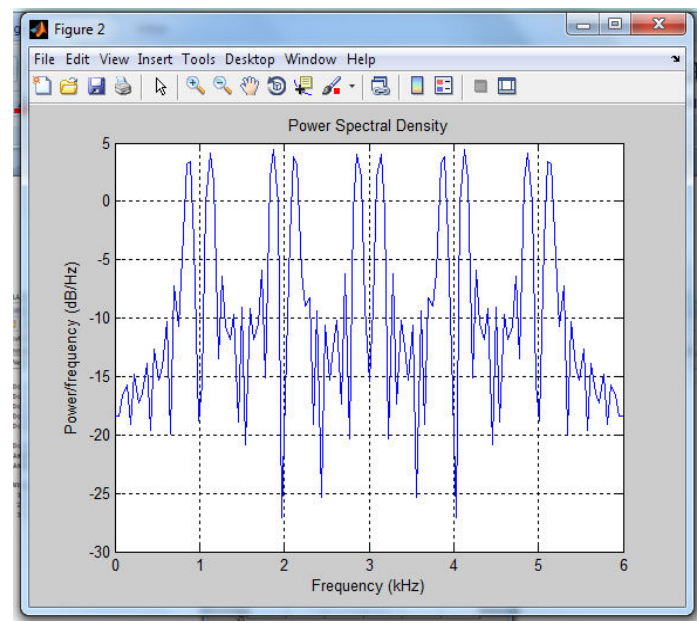

Figure 4 Spectral Efficiency of Four Primary Users where third user spectrum assigned to Secondary user

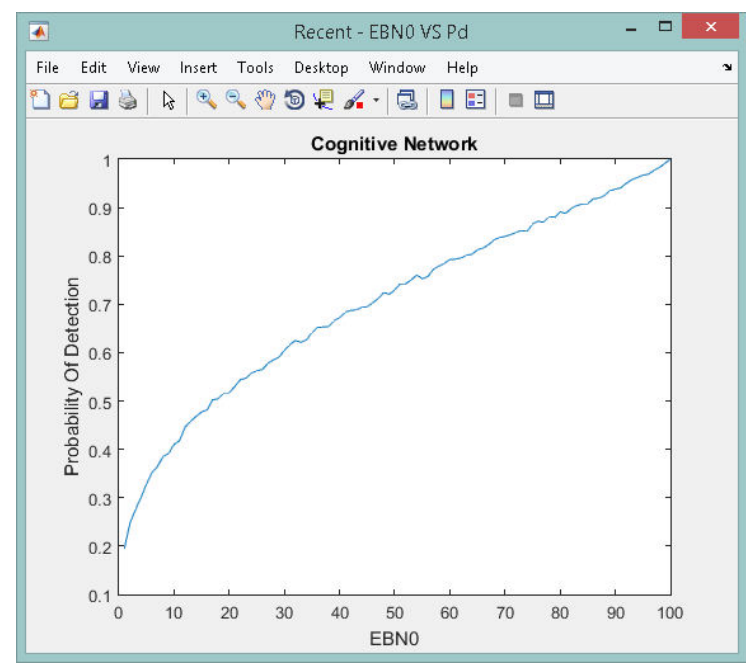

Figure 5 Probability of Detection of four primary Users and a single secondary user.

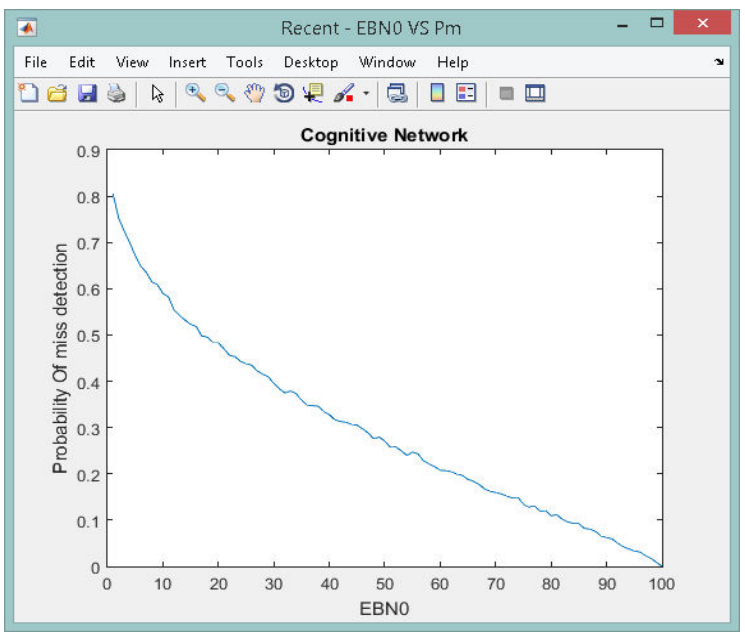

Figure 6 Probability of Miss Detection of four primary Users and a single secondary user. 


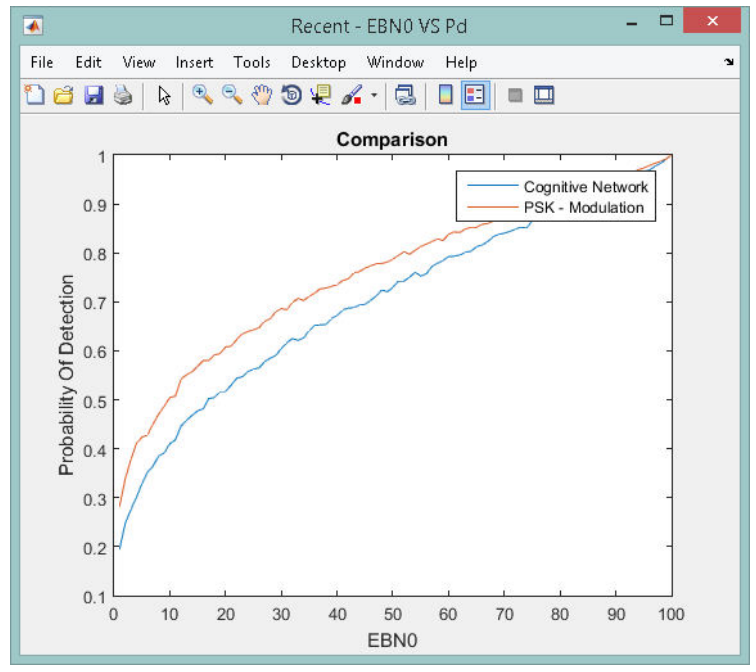

Figure 7 Comparison of Probability of Detection in Cognitive network \& MPSK Modulation

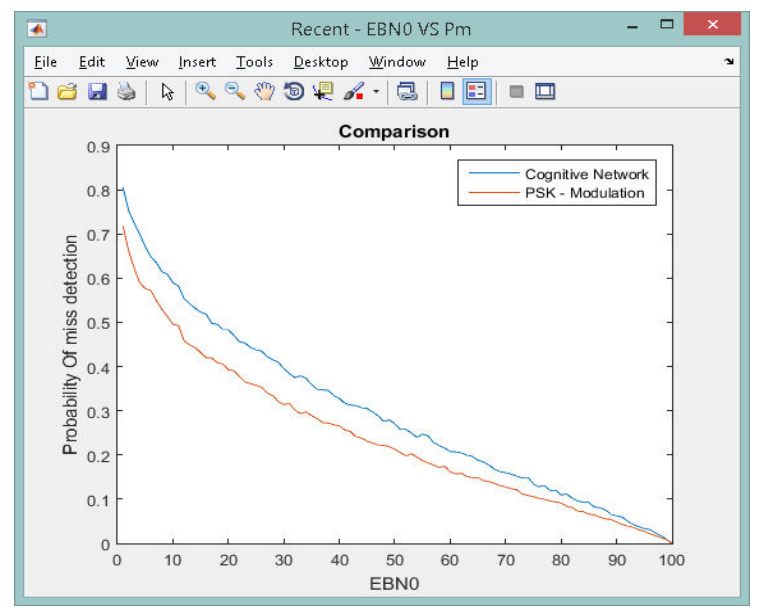

Figure 8 Comparison of Probability of Missed Detection in Cognitive \& MPSK Modulation

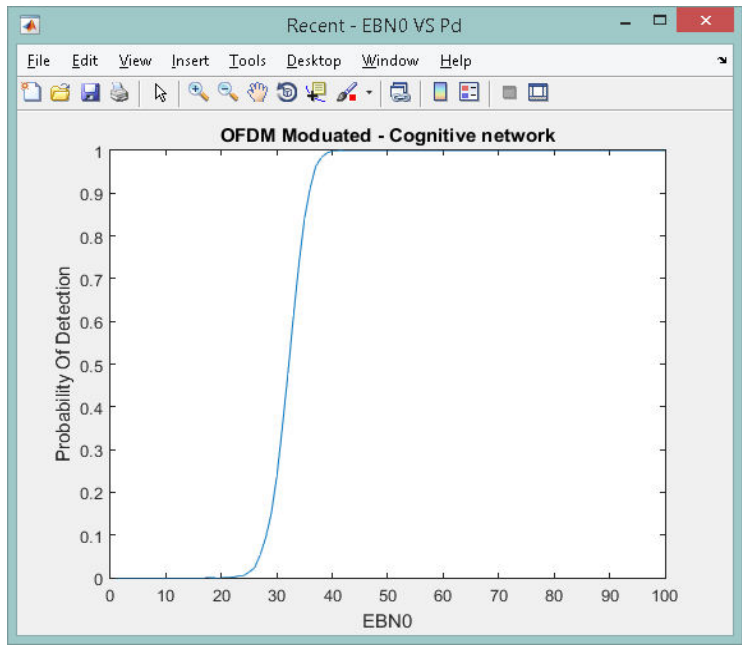

Figure 9 Probability of Detection with OFDM in Cognitive Environment

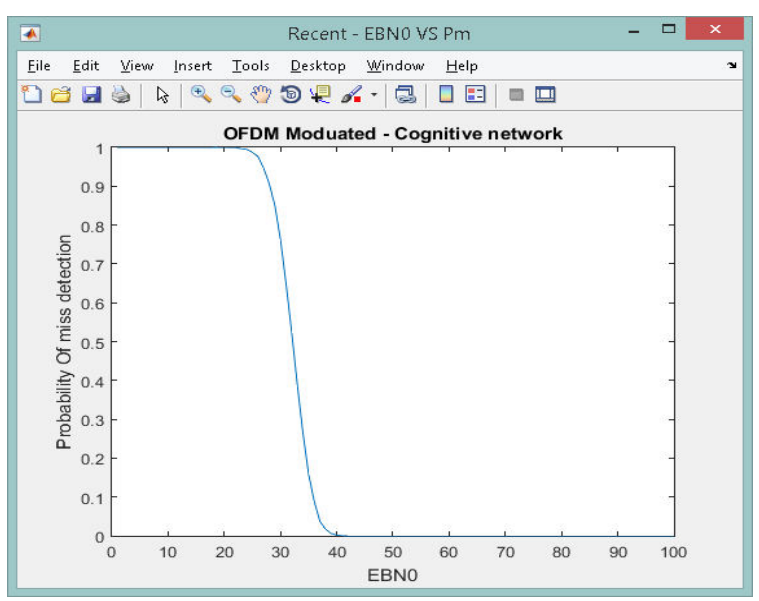

Figure 10 Probability of Missed Detection with OFDM in Cognitive Environment 


\begin{tabular}{|l|l|l|}
\hline SNR & $\begin{array}{l}\mathbf{P}_{\mathbf{d}} \\
\text { Cognitive }\end{array}$ & $\mathbf{P}_{\mathbf{d}}$ (MPSK) \\
\hline $\mathbf{0}$ & $\mathbf{0 . 2 0 1}$ & $\mathbf{0 . 2 9 8}$ \\
\hline $\mathbf{1 0}$ & $\mathbf{0 . 4 1 2}$ & $\mathbf{0 . 4 5 1}$ \\
\hline $\mathbf{2 0}$ & $\mathbf{0 . 5 4 2}$ & $\mathbf{0 . 5 6 7}$ \\
\hline $\mathbf{3 0}$ & $\mathbf{0 . 6 1 2}$ & $\mathbf{0 . 6 4 7}$ \\
\hline $\mathbf{4 0}$ & $\mathbf{0 . 6 7 8}$ & $\mathbf{0 . 7 1 4}$ \\
\hline $\mathbf{5 0}$ & $\mathbf{0 . 7 5 4}$ & $\mathbf{0 . 7 6 2}$ \\
\hline $\mathbf{6 0}$ & $\mathbf{0 . 7 9 8}$ & $\mathbf{0 . 8 1 4}$ \\
\hline $\mathbf{7 0}$ & $\mathbf{0 . 8 5 1}$ & $\mathbf{0 . 8 5 6}$ \\
\hline $\mathbf{8 0}$ & $\mathbf{0 . 8 9 8}$ & $\mathbf{0 . 9 0 1}$ \\
\hline $\mathbf{9 0}$ & $\mathbf{0 . 9 2 7}$ & $\mathbf{0 . 9 3 4}$ \\
\hline $\mathbf{1 0 0}$ & $\mathbf{0 . 9 8 8}$ & $\mathbf{0 . 9 9 7}$ \\
\hline
\end{tabular}

Comparison table of cognitive environment with and without using modulation at different SNR values VI. CONCLUSIONS

In this paper, we built the cognitive radio environment for five primary users along with secondary users. The performance has been investigated in terms of probability of detection and Missed detection and false probability in spectrum sensing technique. The measured detection is compared with the threshold value using energy detection. At SNR value of 60 , the detections are measured 0.798 in cognitive environment \& 0.814 with MPSK Modulation. Whereas, probability of detection value is at peak value with OFDM modulation in which multicarrier is mitigating the effect of intersymbol interference. The result showed that the minimize the spectrum sensing error for a given inequality spectrum sensing constraint. The work is in progress on DWTOFDM implementation in Cognitive Environment.

\section{REFERENCES}

[1] D. Cabric, S. M. Mishra, D. Willkomm, R. W. Brodersen, and A. Wolisz, "A cognitive radio approach for usage of virtual unlicensed spectrum," in Proc. 14th IST Mobile and Wireless Commun. Summit, June 2005.

[2] S. Haykin, "Cognitive radio: brain-empowered wireless communications," IEEE J. Sel. Areas Commun., vol. 23, no. 2, pp. 201-220, 2005.

[3] I. Akyildiz, W. Lee, M. Vuran, and S. Mohanty, "NeXt generation dynamic spectrum access/cognitive radio wireless networks: A survey," Computer Networks, vol. 50, no. 13, pp. 2127-2159, Sep.2006.

[4] I. Mitol, J. and J. Maguire, G.Q., "Cognitive Radio: Making Software Radios more Personal," IEEE Personal Common. Mag., vol.6, no.4, pp.13-18, Aug.1999.

[5] Yonghong Zeng, Ying-Chang Liang, Anh Tuan Hoang, and Rui Zhang. A review on spectrum sensing for cognitive radio: Challenges and solutions. EURASIP Journal on Advances in Signal Processing, 2010:15,2010.

[6] H. Urkowitz, Energy detection of unknown deterministic signals., Proceedings of the IEEE, 55(4):523 - 531, 1967. 
[7] Dong chan oh and yong hwan lee," Energy Detection based spectrum sensing for sensing error minimization in cognitive radio networks", International Journal of comm. Network and information security (IJCNIS) Vol -1, No. 1, April

[8] Ma, G. Y. Li, B.H. Juang. "Signal Processing in Cognitive Radio", Proceedings of the IEEE, vol. 97, pp. 805-823, May 2009.

[9] Pawan Yadav, Rita Mahajan, Energy Detection for Spectrum Sensing In Cognitive Radio Using Simulink ,International Journal of Advanced Research in Electrical, Electronics and Instrumentation Engineering, Vol. 3, Issue 5, may 2014, pp 9367-9372.

[10] Mansi Subhedar and Gajanan Birajdar, spectrum sensing techniques in cognitive radio networks: a survey, international journal of next-generation networks (ijngn) vol.3, no.2, june 2011 pp 37-51.

[11] Y.C Liang, Y Zeng EC Y Peh and A T Hoang, "Sensing throughput tradeoff for Coginitive Radio Networks", IEEE Transaction on Wireless Comm Vol 7, No.11, PP4502-4507, Nov 2008.

[12] Md Shamim Hassain, md Ibrahim Abdullah, Md Alamgir Hossain, " Energy Detection Performance of spectrum sensing in cognitive radio, Int Journal Information Technology and computer science, 2012, PP 11-17.

[13] S.P Herath, N Rajathena and C Tellanbura, "Unified Approach for Energy Detection of unknown deterministic Signal in cognitive radio over fading channels", IEEE Int. Conf on Communication workshop 2009, ICC workshop 2009, PP 1-5.

[14] D. Cabric, S. M. Mishra, and R. W. Brodersen, "Implementation issues in spectrum sensing for cognitive radios," in Proc. Asilomar Conf. Signals, Systems, Computers, Nov. 2004, vol. 1, pp. 772-776.

[15] Prakasam, P. \& Madheswaran, M. (2008). M-ary Shift Keying Modulation Scheme Identification Algorithm Using Wavelet Transform and Higher Order Statistical Moments. Journal of Applied Science 8(1), pages 112-119, 2008.

[16] S.Ghorpade,S.V.Sankpal, "Behaviour of ofdm system using matlab simulation", International Journal Of Advanced computer research (issn (print):2249-7277 issn (online):2277-7970) volume-3 number-2 issue-10 june-2013. 